\title{
New probe for the improvement of the Spatial Resolution in total-body PET (PROScRiPT)
}

\author{
A. $\operatorname{Ros}^{1}$, L. Barrientos ${ }^{1}$, M. Borja-Lloret ${ }^{1}$, J.V. Casaña ${ }^{1}$, E. Muñoz ${ }^{1}$, J. Roser $^{1}$, J.M. Udías ${ }^{2}$, \\ R. Viegas ${ }^{1}$ and G. Llosá ${ }^{1}$ \\ ${ }^{1}$ Instituto de Física Corpuscular (CSIC / UVEG), Paterna, Spain \\ ${ }^{2}$ Grupo de Física Nuclear, EMFTEL, IPARCOS, Universidad Complutense de Madrid, \\ CEI Moncloa, Madrid, Spain
}

\begin{abstract}
In recent decades, PET scanners have been widely used for diagnosis and treatment monitoring in nuclear medicine. The continuous effort of the scientific community has led to improvements in scanner performance. Total-body PET is one of the latest upgrades in PET scanners. These kinds of scanners are able to scan the whole body of the patient with a single bed position, since the scanner tube is long enough for the patient to fit inside. While these scanners show unprecedented efficiency and extended field-of-view, a drawback is their low spatial resolution compared to dedicated scanners. In order to improve the spatial resolution of specific areas when measuring with a total-body PET scanner, the IRIS group at IFIC-Valencia is developing a probe. The proposed setup of the probe contains a monolithic scintillation crystal and a SiPM. The signal of the probe is read out by a TOFPET2 ASIC from PETsys, which has shown good performance for PET in terms of spatial and time resolutions. Furthermore, the PETsys technology generates a trigger signal that will be used to time synchronise the probe and the scanner. The proof-of-concept of the probe will be tested in a Preclinical Super Argus PET/CT scanner for small animals located at IFIC. Preliminary simulations of the scanner and the probe under ideal conditions show a slight improvement in the position reconstruction compared to the data obtained with the scanner, therefore we expect a considerable improvement when using the probe in a total-body PET scanner. Characterisation tests of the probe have been performed with a ${ }^{22} \mathrm{Na}$ point-like source, obtaining an energy resolution of $9.09 \%$ for the $511 \mathrm{keV}$ energy peak and a temporal resolution of 619 ps after time walk correction. The next step of the project is to test the probe measuring in temporal coincidence with the scanner.
\end{abstract}

Index Terms-SiPMs, scintillation crystals, molecular imaging, PET/CT, total-body imaging

\section{INTRODUCTION}

$\mathbf{T}$ HE development of new PET (Positron Emission Tomography) scanners with improved performance is still an important line of research in nuclear medicine. One current line of research is the study of a total-body scanner, the development of which would represent an improvement on the current features of PET scanners [1]. Currently, the typical spatial resolution of reconstructed PET images is between 4 and $6 \mathrm{~mm}$ [2]. The limitation in spatial resolution is a result of the detector module design, the positron range and the noncollinearity of the detected photons [3]. Using smaller detector modules in PET scanners would improve the spatial resolution

This work was supported by grant number SEJIGENT/2019/14. Corresponding author: arosgar@ific.uv.es. but would worsen the sensitivity [4]. For this reason there have been studies suggesting the use of PET probes. Such probes would improve spatial resolution without compromising the scanner sensitivity. The use of a PET probe has been studied in several research groups with a view to improving the spatial resolution: theoretical studies based on Monte Carlo simulations [5], hardware studies using silicon detectors for the PET probe [6] and hardware studies using PsPMT for the PET probe [7]. Furthermore, there are studies of small PET modules with scintillation crystals and SiPM that could be used in PET probes [8]. A PET probe consists of a small detector that operates in temporal correlation with a PET scanner (by means of the scanner electronics or with signal post-processing). In this way, one of the annihilation photons interacts with the PET scanner and the other one with the probe, which has much better spatial resolution than the scanner. The fact that the PET probe can be positioned in the whole field-of-view (FOV) of the PET scanner is of particular interest since the probe increases the spatial resolution of a specific region/lesion of the patient's body $[9,10,11]$. Advances in recent years in commercial data acquisition electronics for PET with high energetic and temporal resolution capabilities has led to the possibility of a new design and implementation for PET probes. The suitability of a PET probe based on scintillation crystals and silicon photomultipliers ( $\mathrm{SiPM})$ is the focus of this study. The functioning of the probe was tested in a Preclinical Super Argus PET/CT scanner for small animals.

\section{Materials AND Methods}

\section{A. Small Animal PET/CT}

The Sedecal Preclinical Super Argus PET/CT is a 4 ring scanner with 24 modules per ring (see Fig. 1a). Each module is composed of a $13 \times 13$ pixel array crystal in a phoswich fashion (LYSO and GSO). The scanner has 1.25 ns nominal time resolution, $25 \%$ nominal energy resolution and $1 \mathrm{~mm}$ nominal spatial resolution in the centre of the FOV. TTL signals of up to $3 \mathrm{~V}$ can be injected via a gating input socket and the data of the injected signal are stored and correlated with the timing of the stored data of the PET scanner. The measured data are singles (raw data) with $40 \mathrm{~ns}$ precision. Calibration measurements with a ${ }^{22} \mathrm{Na}$ point-like source were carried out in order to test the reconstruction code and to determine the 
(a)
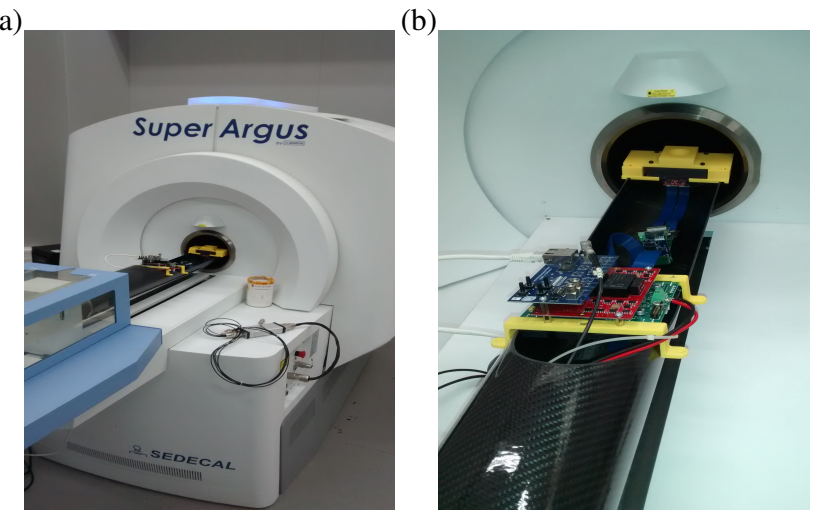

Fig. 1: (a) Super Argus 4R and probe. (b) Detail of the probe.

spatial resolution available with the system. A test with the point-like source in 4 different positions $20 \mathrm{~mm}$ apart along the central axis of the tube (z-axis, coronal plane) was carried out. Additionally, measurements with the point-like source in two positions $10 \mathrm{~mm}$ apart was made, the first with the source at the centre of the FOV and the second with the source 10 $\mathrm{mm}$ above the first (y-axis, transverse plane).

\section{B. Simulations}

GATEv8.2 simulations of the probe and the Preclinical Super Argus PET/CT were performed in order to study the feasibility of the PROScRiPT project. The chosen physics list for the simulation was QGSP_BERT_EMV. The simulation was an ideal simulation; optical photons, reflective material between pixelated crystals, photodetectors and electronics were not simulated The simulated setup consisted of a PET with 4 rings and 24 detector modules per ring, with each module containing an array of $13 \times 13$ scintillation crystals (GSO and LYSO phoswich). The probe, consisting of a continuous scintillation crystal (LYSO) of $25.8 \times 25.8 \times 5 \mathrm{~mm}^{3}$, was positioned at $4 \mathrm{~mm}$ from a small Derenzo-like phantom (a water cylinder with hot-spots) which was in turn positioned in the centre of the FOV of the scanner (see Fig. 2a). The small Derenzo-like hot-spot phantom, contains an activity concentration of 0.108 $\mathrm{mCi} / \mathrm{cc}$ and has several inserts measuring 4.8, 4.0, 3.2, 2.4, 1.6 and $1.2 \mathrm{~mm}$ in diameter. The coincidence time window for the simulation was set to $2 \mathrm{~ns}$. A reconstructed image of the Derenzo-like phantom was obtained for three cases: events in which there were temporal coincidences between any detector in the setup, events in which there were temporal coincidences between the scanner detectors and events in which at least one of the detectors involved in the temporal coincidence was the probe.

\section{The probe}

Regarding the hardware, the probe is being read out by the TOFPET2 ASIC kit from PETsys, a commercial time-offlight PET system. The system provides the digitalised signal of 64 channels per detector and has a high time resolution (time binning $20 \mathrm{ps)} \mathrm{[11].} \mathrm{The} \mathrm{probe} \mathrm{is} \mathrm{made} \mathrm{of} \mathrm{a} \mathrm{SiPM}$ and a scintillation crystal. The size of the crystal $\left(\mathrm{CeBr}_{3}\right)$
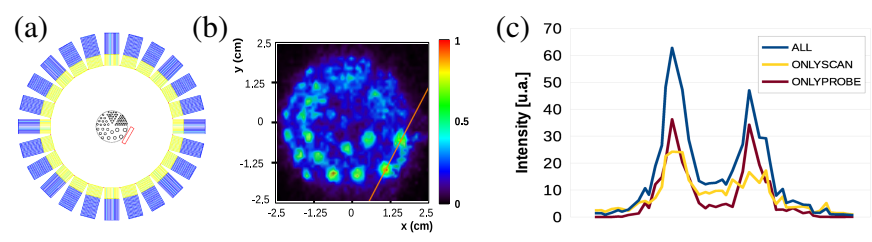

Fig. 2: GATEv8.2 simulations of the probe and the Preclinical Super Argus PET/CT. (a) Gate simulation setup. (b) Source reconstruction. (c) Source reconstruction profiles.

is $25.8 \times 25.8 \times 5 \mathrm{~mm}^{3}$. The SiPMs used for the probe are model S14161-3050HS-08 of Hamamatsu with 64 channels $\left(3 \times 3 \mathrm{~mm}^{2}\right.$ and pixel pitch $\left.50 \mu \mathrm{m}\right)$. All the tests were carried out at a controlled temperature with a maximum variation of three degrees celsius. A set of measurements with two detector modules (DM1 and DM2) were done in order to calibrate the chosen system (see Fig. 3). The measurements were carried out for a distance of $90 \mathrm{~mm}$ between modules and a ${ }^{22} \mathrm{Na}$ point-like source positioned equidistantly between them. The TOFPET2 ASIC has three thresholds [12] (time thresholds (vth_t1 and vth_t2) and energy threshold vth_e) that have been characterised for our setup. Energy and time resolution were measured for different combinations of the threshold values. In order to estimate the best temporal resolution achievable with the system, time-walk corrections (TWC) were applied to the data [13] [14]. Furthermore, to estimate the spatial resolution and test the reconstruction code, measurements with the pointlike source in nine different positions separated by $6.45 \mathrm{~mm}$ were performed. The TOFPET2 ASIC kit has the ability to generate square pulses (with different periods, lengths and total times) that can be configured by the user. The rising times of the generated pulses are stored together with the hits detected by the SiPMs. This signal has been used in order to synchronise the data measured with PETsys and the scanner.

\section{Probe and the scanner measurements}

In order to be able to measure with the probe and the scanner it is necessary to synchronise them and to determine

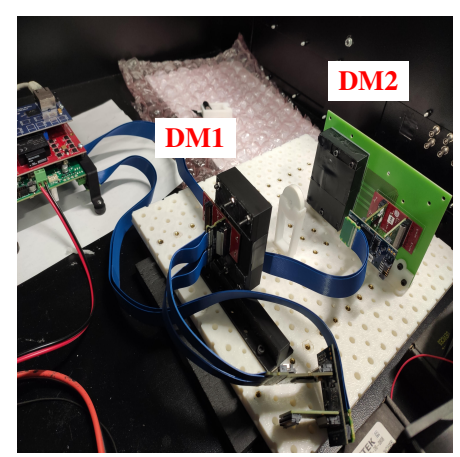

Fig. 3: Probe calibration setup. Detector modules 1 (DM1) and 2 (DM2) are composed of a SiPM (S14161-3050HS-08, Hamamatsu) and a monolithic $\mathrm{CeBr}_{3}$ crystals. 
the position of the probe within the scanner. For the synchronisation the output trigger signal generated by PETsys has been used. A series of four trigger signals were generated every time a measurement was performed. The first, second and forth trigger signals lasted $30 \mathrm{~s}$. The data measured during these triggers were not used for the reconstruction since these triggers were only used to check the integrity of the generated signal. The third trigger, $1800 \mathrm{~s}$ long, and the data measured during this time with the probe and the scanner, are used for the reconstruction. A dead-time of around $80 \mathrm{~ns}$ has been measured between the generation of one trigger signal and the next. The trigger signal generated by PETsys was injected into a TTL input port of the Super Argus 4R scanner. The scanner reads out the input trigger signal and stores the rising and falling time of the pulses, relating their timing with the internal time of the scanner. The position of the probe in the scanner was determined based on the position of the reconstructed ${ }^{22} \mathrm{Na}$ point-like source and the fact that the probe and the source were mounted in a custom holder of known dimensions.

\section{RESULTS}

All the images were reconstructed using an ideal MLEM code (no sensitivy matrix or corrections) and all the reconstructions were stopped at the $5^{\text {th }}$ iteration.

\section{A. Simulation results}

Fig. 2b shows a detail of the superimposed reconstructed Derenzo-like phantom for the three cases. The profiles shown in Fig. 2c correspond to the data of the slice represented by the orange line in Fig. 2b. It can be appreciated that the spatial resolution has slightly improved for the case in which the coincidences occurred between the probe and the scanner with respect to the other two cases.

\section{B. Probe characterisation}

Table I shows the tested values for the characteristic TOFPET2 ASIC thresholds, the energy resolution $\left(E_{R}\right)$ and the coincidence time resolution (CTR) obtained for each case. The timing was calculated using two different methods: (i) selecting the time of the most energetic hit $\left(\mathrm{CTR}_{\mathrm{E}}\right)$ and (ii) calculating the average time for the first five hits $\left(\mathrm{CTR}_{\mathrm{av}}\right)$. A clear improvement of the time resolution is obtained when using the averaged time for the five first hits.

The parameters that led to the best results were: threshold vth_t1, vth_t2 and vth_e set to 20,5 , and 5 respectively. The best value for the energy resolution was $9.09 \%$ for the 511 $\mathrm{keV}$ energy peak. In order to determine the best CTR, the average of the five first hits was calculated, following which the energy was filtered between 450 and $600 \mathrm{keV}$ and, after applying time-walk corrections, a CTR of 619 ps was found. Fig. 4 shows the time difference (ps) versus energy (keV) for the studied detector modules (DM1 and DM2) before and after time-walk correction (Fig. $4 \mathrm{a}$ and $4 \mathrm{~b}$ show the data with

\section{TABLE I:}

Characterisation of the TOFPET2 ASIC thresholds. Values of energy resolution at $511 \mathrm{keV}$ peak and CTR (calculated by two different ways) are shown.

\begin{tabular}{||l||c|c|c||c|c|c||c|c|c|c|c|c||}
\hline Vth_t $_{1}$ & 20 & 15 & 10 & 10 & 10 & 10 & 10 & 10 & 10 & 10 & 10 & 10 \\
\hline Vth_t $_{2}$ & 5 & 5 & 5 & 20 & 18 & 15 & 10 & 10 & 10 & 10 & 10 & 10 \\
\hline Vth_e & 5 & 5 & 5 & 5 & 5 & 5 & 10 & 9 & 8 & 7 & 6 & 5 \\
\hline $\begin{array}{l}\mathrm{E}_{\mathrm{R}} \\
511 \mathrm{keV}\end{array}$ & 9.09 & 9.43 & 9.72 & 9.55 & 9.79 & 10.25 & 10.83 & 10.64 & 10.56 & 10.84 & 10.45 & 10.61 \\
\hline $\begin{array}{l}\text { CTR } \\
\text { (ns) }\end{array}$ & 2.61 & 2.69 & 2.61 & 2.40 & 2.44 & 2.66 & 2.37 & 2.51 & 2.66 & 2.40 & 2.77 & 3.03 \\
\hline $\begin{array}{l}\text { CTR } \\
\text { (ns) }\end{array}$ & 0.88 & 0.82 & 0.80 & 1.07 & 0.93 & 0.83 & 0.80 & 0.78 & 0.80 & 0.81 & 0.82 & 0.85 \\
\hline
\end{tabular}
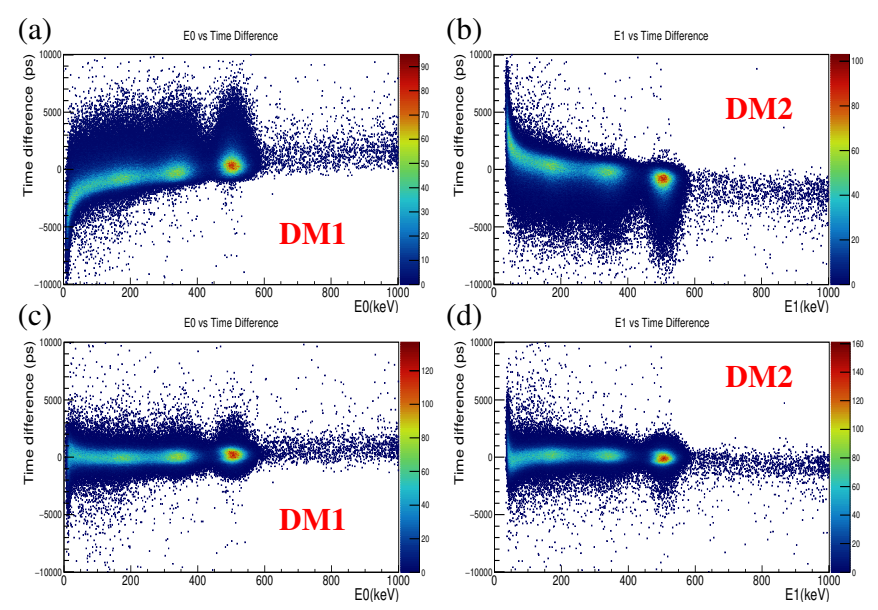

Fig. 4: Time difference (ps) versus energy (keV) for the studied detector modules.

no time-walk correction and Fig. $4 \mathrm{c}$ and $4 \mathrm{~d}$ show the data with time-walk correction). This shows good agreement with the value of 660 ps obtained by Lamprou et al., using two monolithic scintillation crystals $\left(50 \times 50 \times 15 \mathrm{~mm}^{3}\right)$ optically coupled to $8 \times 8 \mathrm{SiPMs}$ arrays (ON-Semi, J-series mode) [15].

Fig. 5a shows a schematic of the nine different positions in which the ${ }^{22} \mathrm{Na}$ point-like source was positioned between DM1 and DM2. The reconstructed positions can be seen in Fig. $5 b$. Furthermore, the horizontal profiles for positions $[1,2,3]$, $[4,5,6]$ and $[7,8,9]$ are shown in Fig. 5c, 5d and 5e respectively. A value of $1.29 \mathrm{~mm}$ was obtained for the FWHM for the central position (position 5).

\section{Scanner characterisation}

A ${ }^{22}$ Na point-like source was used to perform measurements with the Sedecal Preclinical Super Argus PET in order to estimate the spatial resolution achievable with the scanner and the imaging reconstruction code used in the present research. Fig. 6 shows the reconstructed position of the point-like source in four different positions (20 mm apart) along the centre of the scanner tube (z-axis). Fig. 6a shows the reconstructed positions in the transverse plane while Fig. 6b shows the same data for the sagittal plane. Fig. $6 \mathrm{c}$ corresponds to the profile through the $\mathrm{x}$-axis for all the $\mathrm{z}$-voxels in the FOV and Fig. $6 \mathrm{~d}$ corresponds to the profile through the $y$-axis, summing five neighbour voxels in the $\mathrm{x}$ and $\mathrm{z}$-axis. The difference in the 
(a)

(c)

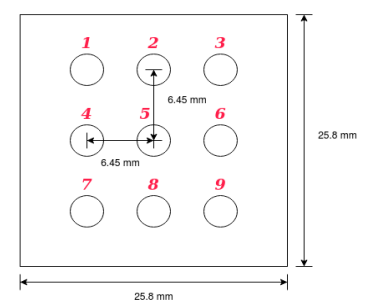

(b)

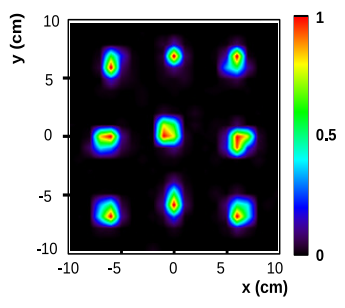

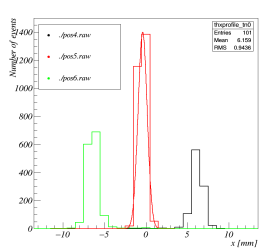

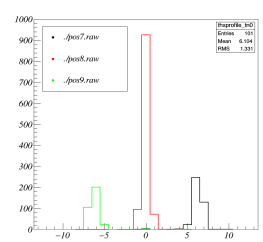

Fig. 5: ${ }^{22} \mathrm{Na}$ point-like source position reconstruction for nine positions. (a) Positioning of the ${ }^{22} \mathrm{Na}$ point-like source. (b) Reconstructed data. (c) Profiles of the reconstructed data.

numbers of events that is visible in Fig. $6 \mathrm{~d}$ is due to the lack of a sensitivy matrix in the reconstruction code. A FWHM of $1.94 \mathrm{~mm}$ was obtained for the reconstructed distribution for the position at around $40 \mathrm{~mm}$, in the centre of the FOV, as can be seen from the number of events measured in that position.

Fig. 7 shows the reconstructed ${ }^{22} \mathrm{Na}$ point-like source position in the centre of the scanner and $10 \mathrm{~mm}$ above. The images for the transverse and sagittal planes are shown in Fig. 7a and $7 \mathrm{~b}$, respectively. Fig. 7c shows the profile of the data through the $\mathrm{x}$-axis summing five neighbour voxels in the $\mathrm{y}$ and $\mathrm{z}$ axis. Fig. $7 d$ shows the profile of the data through the $y$-axis summing five neighbour voxels in the $\mathrm{x}$ and $\mathrm{z}$-axis. In this case, Fig. $7 \mathrm{~d}$ also clearly shows the difference in the number of events measured outside the central area of the FOV, but it is still possible to correctly determine the position of the source.

\section{Probe and scanner measurement}

Fig. 8 shows two reconstructed images for a ${ }^{22} \mathrm{Na}$ pointlike source. The first one corresponds to the case in which data was stored when a temporal coincidence occurs between the detectors of the scanner (scanner-scanner) and between the scanner detectors and the probe (scanner-probe). In the second case, only the temporal coincidences of the type scannerprobe for the case in which the coincidences occured between the probe and the detector of the scanner facing the probe were considered. In both cases the coincidence time window was set to $100 \mathrm{~ns}$ and an energy filter from $400 \mathrm{keV}$ to $660 \mathrm{keV}$ was applied to the data. In Fig. $8 \mathrm{a}$ and $8 \mathrm{~b}$ the upper point corresponds to the first case while the lower point corresponds to the second case. A mispositioning of the reconstructed point-like source can be appreciated between the two cases. The normalised profiles for the reconstructed source are shown in Fig. 8c and 8d. The black distribution corresponds to the data with coincidences of the type scannerscanner and scanner-probe and the red distribution to the data with coincidences of the type scanner-probe. Fig. 8 shows

(a)

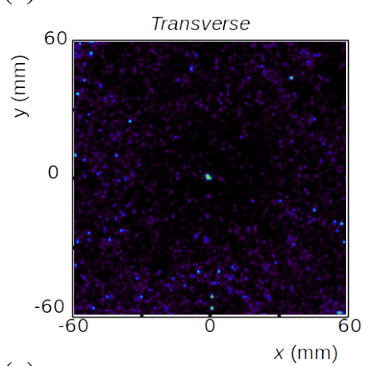

(c)

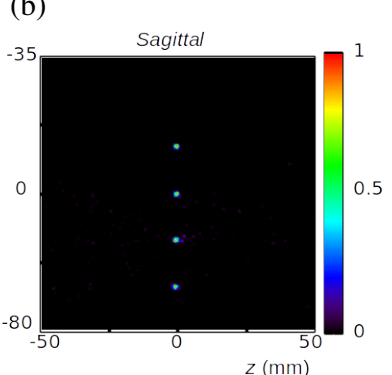

(d)
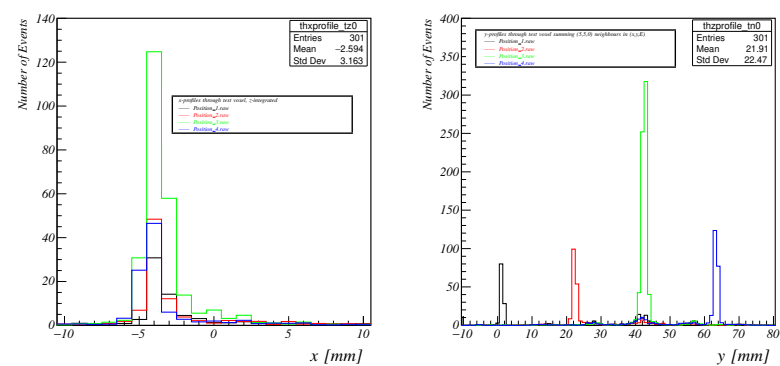

Fig. 6: Reconstructed position (transverse (a) and sagittal (b) planes) of the point-like source in four different positions (20 mm apart) along the centre of the scanner tube (z-axis). Profiles of the reconstructed data through the x-axis (c) and y-axis (d). (a)

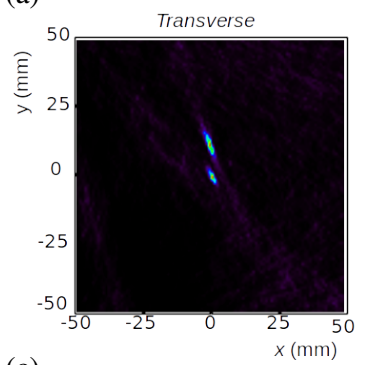

(c)

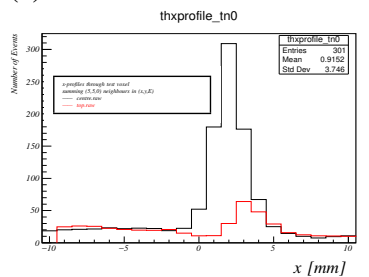

(b)

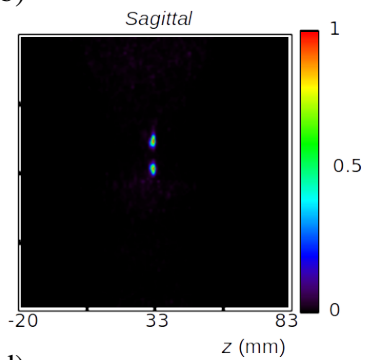

(d)

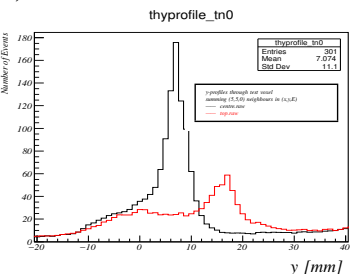

Fig. 7: Reconstructed ${ }^{22} \mathrm{Na}$ point-like source position (transverse (a) and sagittal (b) planes) in the centre of the scanner and $10 \mathrm{~mm}$ above. Profiles of the reconstructed data through the $\mathrm{x}$-axis (c) and y-axis (d).

that it is possible to reconstruct the ${ }^{22} \mathrm{Na}$ point-like source distribution for both cases.

\section{DISCUSSION AND CONCLUSION}

The simulation results show a slight improvement in the spatial resolution while measuring with the probe and the scanner. Small animal PETs have high spatial resolution, and 
(a)

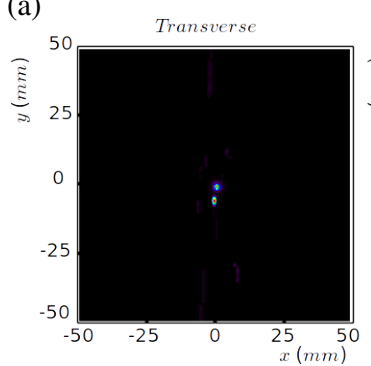

(c)

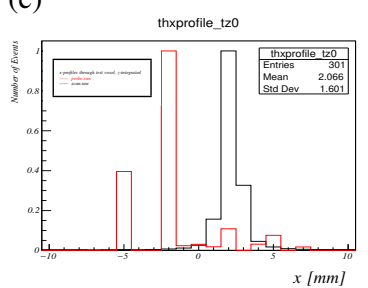

(b)

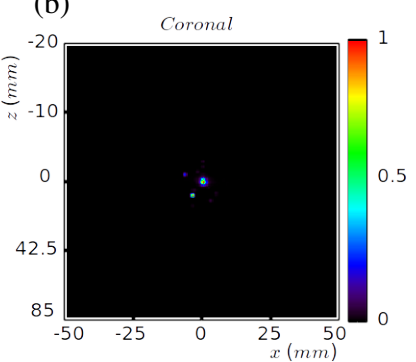

(d)

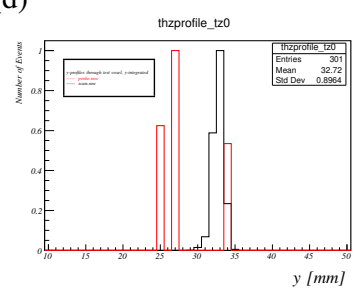

Fig. 8: Two reconstructed images for the ${ }^{22} \mathrm{Na}$ point-like source. The upper point corresponds to the cases scannerscanner and scanner-probe and the lower point to the case scanner-probe. Transverse and sagittal planes are shown in (a) and (b), respectively. Profiles of the reconstructed data through the $\mathrm{x}$-axis and $\mathrm{y}$-axis are shown in (c) and (d), respectively.

the spatial resolution in TBP scanners in the future.

\section{REFERENCES}

[1] Cherry, Simon R et al. et al., Journal of Nucl. Med., Society of Nuclear Medicine vol. 59, 13-12, 2018.

[2] Zanzonico P.B. et al., Essentials of in vivo biomedical imaging, CRC Press, Boca Raton Florida U.S.A., 2015.

[3] Zhou, J., et al., Phys. Med. Biol., vol. 54, no. 17, 5193-5208, 2009.

[4] Park, S-J. et al., Phys. Med. Biol., vol. 52, no. 15, 4653-4677, 2007.

[5] Clinthorne N. et al., Physics Procedia, vol. 37, 1488-1496, 2012.

[6] Wu, H. et al., Journal of Nucl. Med., Society of Nuclear Medicine, vol. 49, no. 10, 1668-1676, 2008.

[7] Jiang J. et al., IEEE transactions on medical imaging, vol. 39, no. 11, 3268-3277, 2019.

[8] Song, T. Y. et al., Phys. Med. Biol., vol. 55, no. 9, 2573- 2587, 2010.

[9] Grkovski, Milan. et al., Nucl. Inst. and Meth. A, vol. 788, 86-94, 2015.

[10] Brzeziński, K. et al., JINST, vol. 11, P09016, 2016.

[11] Bugalho R. et al., JINST, vol. 14, no. 03, 2019.

[12] Schug D. et al., IEEE Transactions on Radiation and Plasma Medical Sciences, vol. 3, no. 04, 444-453, 2018.

[13] Poulson D. et al., Space Telescopes and Instrumentation 2020: Ultraviolet to Gamma Ray, vol. 11444, 114446D, 2020.

[14] Hueso-Gonzalez F. et al., JINST, vol. 9, no. 05, P05002, 2014.

[15] Lamprou E. et al., Phys. Med., vol. 70, no. 70, 10-18, 2020. therefore it is expected that using the probe in a total-body PET scanner, in which the intrinsic spatial resolution is worse, would improve the resolution notably. The values obtained in the characterisation of the probe suggest that it is a good candidate for the proof-of-concept.

The measured value of the FWHM measured with the probe setup for the central position is promising although a resolution of approximately $1 \mathrm{~mm}$ would be desirable. Tests with different scintillation crystals, both monolithic and pixelated, and with different types of SiPM should be perfomed. Furthermore, the obtained value for the CTR, of the order of ps, suggests that the TOFPET ASIC kit is a good candidate to read out the probe signal. Regarding the trigger signal generated by the TOFPET ASIC kit, the signals generated have been shown to be sufficiently accurate for the probe synchronisation. The reconstructed data using the Super Argus 4R scanner show that the reconstruction code is acceptable for the initial stages of this project, although it should be improved for the final probe prototype.

The results obtained for the measurement of the point-like source with the scanner and the probe show that the synchronisation of the probe and the scanner was succesful using the signal trigger of the TOFPET ASIC kit and the input gating signal of the scanner. The mispositioning of the reconstructed point-like source for the scanner-probe case suggests that the methodology used to determine the positioning of the probe within the scanner must be improved. The lack of a sensitivity matrix in the reconstruction code would also have influenced the mispositioning of the probe. Overall, the results obtained so far in the PROScRiPT project are sufficiently promising to suggest that the positioning and synchronisation of a probe within a PET scanner could realistically be used to improve 